\title{
Telomeres and age-related disease: how telomere biology informs clinical paradigms
}

\author{
Mary Armanios \\ Department of Oncology, Sidney Kimmel Comprehensive Cancer Center, and McKusick-Nathans Institute of Genetic Medicine, \\ Johns Hopkins University School of Medicine, Baltimore, Maryland, USA.
}

\begin{abstract}
Telomere length shortens with age and predicts the onset of replicative senescence. Recently, short telomeres have been linked to the etiology of degenerative diseases such as idiopathic pulmonary fibrosis, bone marrow failure, and cryptogenic liver cirrhosis. These disorders have recognizable clinical manifestations, and the telomere defect explains their genetics and informs the approach to their treatment. Here, I review how telomere biology has become intimately connected to clinical paradigms both for understanding pathophysiology and for individualizing therapy decisions. I also critically examine nuances of interpreting telomere length measurement in clinical studies.
\end{abstract}

Understanding basic biological mechanisms holds the potential to advance clinical paradigms. The emerging impact of telomerase and telomere biology in medicine provides a clear example of this promise. Research in this area was initially sparked by fundamental questions about how genomes are protected at chromosome ends, and focused on curiosity-driven questions in maize, yeast, and protozoa (1). These highly conserved molecular mechanisms have now led to unforeseen benefits for understanding idiopathic disease and have opened a new area of translational research. Here I review the trajectory of the evolving role of telomere biology in clinical paradigms and highlight how it has become central to understanding the pathophysiology of age-dependent disorders as well as for informing new approaches to their treatment.

\section{Telomeres and telomerase}

Telomeres define the ends of linear chromosomes. They are made up of repetitive DNA sequences that are bound by specialized proteins. The human telomeric DNA sequence is a tandem repeat of TTAGGG that extends several kilobases (a mean of 10 kilobases in umbilical cord blood) (2-4). The telomere-binding complex of proteins, known as shelterin, together with telomere DNA, functions as a dynamic unit that protects chromosome ends from being recognized as broken DNA, thus preventing their degradation and participation in fusion events (5). Telomeres are therefore essential for the maintenance of genomic integrity.

Telomerase is the specialized polymerase that synthesizes new telomere repeats $(6,7)$. It offsets the shortening that normally occurs with cell division since the replication machinery does not copy fully to the ends. Telomerase has two essential core components, the telomerase reverse transcriptase (TERT) and the telomerase RNA (TR), the latter of which provides the template for telomere repeat addition (8-10). In human cells, telomerase is the primary mechanism by which telomeric DNA is synthesized de novo. As will be discussed, mutations in the TERT and TR genes are considered the most common cause of inherited human telomere-mediated disease (11). Even with mild perturbations in telomerase activity, telomere length homeostasis is disturbed and manifests in what has become recognized as a discrete syndrome complex, which recapitulates age-dependent disease processes

Conflict of interest: The author has declared that no conflict of interest exists. Citation for this article: J Clin Invest. 2013;123(3):996-1002. doi:10.1172/JCI66370.
$(12,13)$. As such, these mutations and their clinical consequences are the primary focus of this Review.

\section{Telomere length is heritable}

Telomeres have long been linked to processes of cellular aging. Since the 1990s it has been known that telomere length predicts the onset of replicative senescence $(14,15)$, a permanent state of cell cycle arrest that primary cells reach after they undergo a finite number of cell divisions (16). The fact that telomeres also shorten in vivo in humans with advancing age made a further compelling case for the idea that telomeres play a role in age-related processes. The evidence reviewed here shows that telomere shortening is sufficient to provoke age-related pathology. Several factors ensure that telomere shortening is a default state in somatic cells. Although telomerase offsets the end-replication problem, its levels are tightly regulated and only a few telomeres are elongated in each cell cycle (17). Therefore, even cells that may be relatively enriched for telomerase activity, such as hematopoietic stem cells, undergo telomere shortening with age (4). The incremental elongation of telomeres by telomerase can also be seen across generations (18). For both humans and mice, the telomere length of parentes determines the telomere length of their offspring (19-21). These observations have further established telomere length as a unique genotype (at times referred to as "the telotype") and as a source of genetic variation across human populations (22).

\section{Senescence and its emerging role in degenerative disease}

When telomeres become critically short, they become dysfunctional and activate a DNA damage response that resembles double-strand breaks (23). The resulting signaling cascade provokes apoptosis and/or a permanent cell cycle arrest that, until recently, has been considered the primary functional consequence of senescence. Cell type-dependent factors determine whether apoptosis, senescence, or a combined phenotype predominates in response to dysfunctional telomeres $(14,24,25)$, although the molecular effectors that discriminate between these pathways are not entirely understood.

Recently, a more complex understanding of the senescence phenotype has been emerging and suggests a closer link to disease mechanisms than was previously appreciated. For example, although senescent cells are quiescent in the cell cycle, for reasons that are not entirely clear, their gene expression profile is 
Telomere syndrome manifestations that overlap with human age-related phenotypes

High-turnover compartments

Hair graying

Hair loss

Nail ridging

Periodontal disease

Thrombocytopenia

Decreased bone marrow cellularity

Immunosenescence

Gastrointestinal intraepithelial lymphocytosis

Increased cancer risk

Chemotherapy intolerance
Low-turnover compartments

Idiopathic pulmonary fibrosis

Emphysema

Liver fibrosis and cirrhosis

Impaired glucose tolerance

Defective insulin secretion

Insulin resistance

Osteoporosis

atric telomere syndrome cases, especially in the setting of dyskeratosis congenita, which was the first genetic disorder to be linked to telomere biology (36-38). Biallelic mutations in the conserved telomere component 1 gene, CTC1, which plays a putative role in telomere lagging strand synthesis, have also been recently implicated in rare autosomal recessive cases that also have predominantly pediatric presentations (39-41). There remains a subset of cases with inherited telomere phenotypes for which the mutant genes are unknown; their identification is the focus of ongoing research.

altered (26). One consequence of this altered gene expression is that senescent cells secrete a predictable profile of cytokines, chemokines, and proteases into culture media, a phenotype known as the senescence-associated secretory phenotype $\operatorname{SASP})(27,28)$. In vivo, the SASP has been hypothesized to play a role in the clearance of damaged cells (29). Telomere dysfunction is furthermore associated with a state of decreased cellular metabolic activity (25, 30). In mice with short telomeres, defective cellular metabolism in the setting of senescence manifests as mitochondrial dysfunction and aberrant $\mathrm{Ca}^{2+}$ signaling that cause insulin secretory defects by pancreatic $\beta$ cells (25). These defects disturb glucose homeostasis in vivo. The fact that cellular senescence is associated with defective signaling and metabolism provides new contexts for understanding mechanisms of degenerative disease with age, particularly because these defects might occur in the absence of overt histopathology (25).

\section{Mutant telomere genes cause premature aging disorders}

The most compelling evidence that telomeres contribute to aging comes from the fact that mutant telomerase and telomere genes cause telomere shortening that manifests in age-related phenotypes (see Telomere syndrome manifestations that overlap with human age-related phenotypes). Because telomere shortening is acquired universally with age, these disorders have a particular relevance for understanding mechanisms of age-related disease. Telomere-mediated disorders show two hallmarks of age-related disease: degenerative organ failure and a cancer-prone state (31). Age-related disease is additionally marked by atherosclerosis; however, premature vascular disease has not been reported and does not, in our experience, seem to be accelerated in individuals with telomere disorders.

Eight genes have been implicated in monogenic telomere disorders (reviewed in ref. 22). The most prevalent are heterozygous mutations in TERT and TR, which cause autosomal dominant disease. The dominant mode of inheritance occurs as a result of the sensitivity of telomere maintenance to telomerase levels, even when only one allele is perturbed $(12,18,32-35)$. Mutations in $T E R T$ and TR usually cause significant morbidity after the reproductive age is reached, and a greater number of offspring are affected as a result of their dominant mode of inheritance. They are thus estimated to be the most prevalent cause of inherited telomere disorders, comprising at least $90 \%$ of cases (11). Mutations in genes encoding the $\mathrm{X}$-linked telomerase accessory component, DKC1, which is essential for human TR stability, and the autosomal shelterin gene, TINF2, explain a significant subset of pedi-

\section{Recognizing a telomerase mutation carrier}

Telomere-mediated disease has diverse presentations that span the age spectrum. Their type, age of onset, and severity depend on the extent of the telomere length defect. In infancy, severe telomere shortening manifests as developmental delay, cerebellar hypoplasia, and immunodeficiency, features that are recognized in the rare Hoyeraal-Hreidarsson syndrome (42). In children and young adults, telomere-mediated disease causes bone marrow failure and at times may be recognized in the mucocutaneous syndrome dyskeratosis congenita, which is defined by a triad of mucocutaneous features - skin hyperpigmentation, dystrophic nails, and oral leukoplakia $(33,43,44)$. Telomere-mediated disease manifests in adults as isolated or syndromic clustering of idiopathic pulmonary fibrosis (IPF), liver cirrhosis, and bone marrow failure (31). Mutant TERT and TR genes account for $8 \%-15 \%$ of familial and $1 \%-3 \%$ of sporadic pulmonary fibrosis cases (45-47). Because IPF affects at least 100,000 individuals in the United States, it is considered the most prevalent manifestation of the telomere disorders (11). An individual who carries a telomerase mutation will therefore most frequently be clinically recognized as an adult with familial pulmonary fibrosis. Adult-onset telomere disease may rarely also manifest as sporadic or familial myelodysplastic syndrome or acute myeloid leukemia (48-50). The co-occurrence of IPF and bone marrow failure within a single family is highly predictive for the presence of a germline telomerase defect (51).

Although the manifestations of telomere-mediated disease occur in multiple organs and may appear clinically different, it has been proposed that their shared short telomere length defect unifies them under the umbrella of a single syndrome continuum $(12,22$, $31,45,46,51)$. This molecular classification is significant because the telomere defect is present in the germline of these patients and thus, even when a single presentation predominates, complications that are relevant to managing symptoms and averting complications may arise in other organs. The regrouping of what have historically been considered unrelated disorders provides new clinical insights as these conditions significantly overlap. The consideration of the telomere syndromes as a single spectrum exemplifies how a molecular classification of disease may help explain previously mysterious complications of treatment and refine clinical approaches.

\section{Telomere-mediated disease manifests as stem cell failure in high-turnover tissues}

The clinical manifestations of telomere shortening can be divided into two broad categories: those affecting high-turnover tissues and 

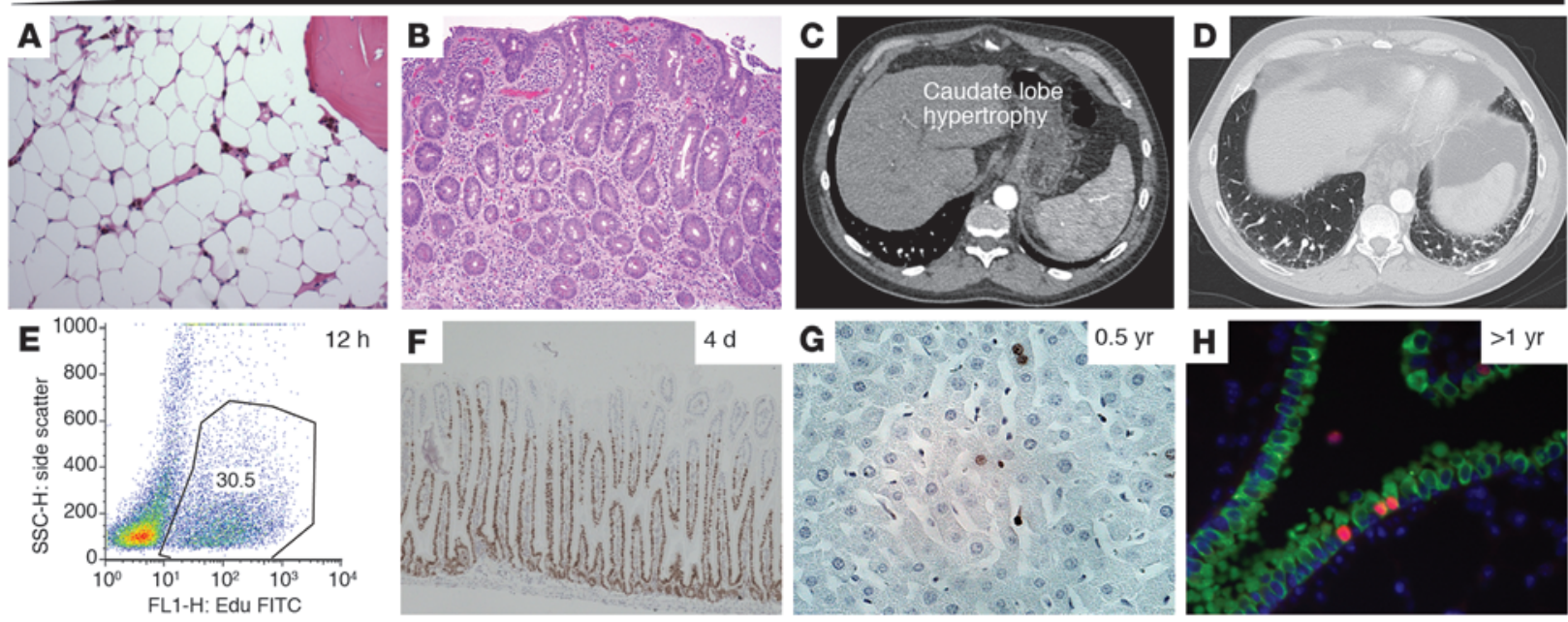

Proliferation rate

\section{Figure 1}

Clinical manifestations of telomere disorders and their onset relative to tissue turnover rate. Shown are representative images of diagnostic histopathology and radiographic studies in patients with telomere-mediated disease (A-D) and 5-ethynyl-2'-deoxyuridine (EdU) incorporation detected in corresponding mouse tissues (E-H). The estimated turnover rate of more than $90 \%$ of cells is indicated for each pair of images. (A) Photomicrograph of a bone marrow biopsy showing an acellular marrow replaced by adipose tissue with only remnants of hematopoiesis, taken from an individual with aplastic anemia. Image reproduced with permission from Annual Reviews of Genomics and Human Genetics (31). (B) Histopathology of a duodenal biopsy from a patient with telomere-mediated enteropathy shows profound villous atrophy. Image reproduced with permission from Aging Cell (53). (C) Abdominal CT scan image from a patient with liver cirrhosis, as evidenced by the nodular liver surface, the caudate lobe hypertrophy, and splenomegaly. (D) Lung windows of a chest CT scan from a carrier of the telomerase mutation show classic basilar honeycombing changes pathognomonic for IPF. (E) Flow cytometry plot of EdU incorporation in the bone marrow after a short (2-hour) pulse, showing that nearly one-third of the cells have undergone division. (F) Immunohistochemistry of intestinal section after a EdU pulse (5 days) shows that nearly all enteric epithelial cells are positively labeled (brown). (G) Brown staining shows EdU-labeled hepatocytes after EdU labeling (14 days). (H) Image of terminal bronchiole shows EdU-positive lung epithelial cells (red) identified by the Clara cell antigen (green) after 14 day label.

those affecting low-turnover tissues. This distinction is important for understanding disease patterns because the high-turnover phenotypes tend to appear first in pediatric populations and represent more severe disease (ref. 51 and Figure 1). For example, telomere syndromes in infancy manifest as severe immunodeficiency, which affects B cells, T cells, and NK cells, coincident with the extraordinary replicative demands on the adaptive immune system during this period of development $(42,52,53)$. Bone marrow phenotypes tend to appear later in children and young adults as isolated cytopenias or aplastic anemia $(43,45,51,54)$. The hematopoietic defects have been studied in animal models and represent a stem cell failure state whereby short telomere length limits both stem cell number and function $(33,51,55-57)$. The telomere-mediated bone marrow failure phenotype is stem cell autonomous because allogeneic stem cell transplantation can reverse this state. The gastrointestinal epithelium, another high-turnover compartment, is also affected in a subset of patients who develop an enteropathy marked by villous blunting that resembles celiac disease (53). These intestinal phenotypes are similarly thought to be caused by stem cell failure that appears as villous atrophy in mice with short telomeres $(18,58)$.

\section{Telomere phenotypes in adults predominantly manifest in slow-turnover tissues}

More commonly, telomere-mediated disease manifests in slowturnover tissues, such as the lung and the liver (Figure 1). These phenotypes frequently appear as de novo adult-onset disease, in contrast to the pediatric presentations of dyskeratosis congenita and related disorders. IPF presents at mean age between 50 and 60 years (range $31-87)(35,45,47,51,59,60)$, and telomere-related cryptogenic liver fibrosis, based on reported cases, presents at a mean of 37 years (range 20-57) $(12,59,61)$. The mechanisms of these adult-onset disorders can also be distinguished in animal models. In contrast to the high-turnover phenotypes that are readily evident in the telomerase knockout mouse, telomere dysfunction in slow-turnover organs serves as the first of multiple acquired "hits" that contribute ultimately to organ failure (Figure 2). For example, mice with short telomeres do not develop de novo lung phenotypes, but acquire them only after chronic injury such as with cigarette smoke (62). Similarly, liver damage is only detected when mice with short telomeres are challenged with carbon tetrachloride (63). In the endocrine pancreas, telomere dysfunction cooperates with genetically induced endoplasmic reticulum stress to cause $\beta$ cell apoptosis and manifest in worsening diabetes severity (25). Therefore, in tissues in which adult cell turnover is minimal, telomere dysfunction disturbs organ homeostasis because of cumulative hits in long-lived cells and eventually culminates in what appears as irreversible adult-onset disease (Figure 2). The cell types responsible for the telomere-induced fibrotic disorders are not known, but it has been hypothesized that these disorders, similar to the telomere-dependent bone marrow and intestinal defects, rep- 


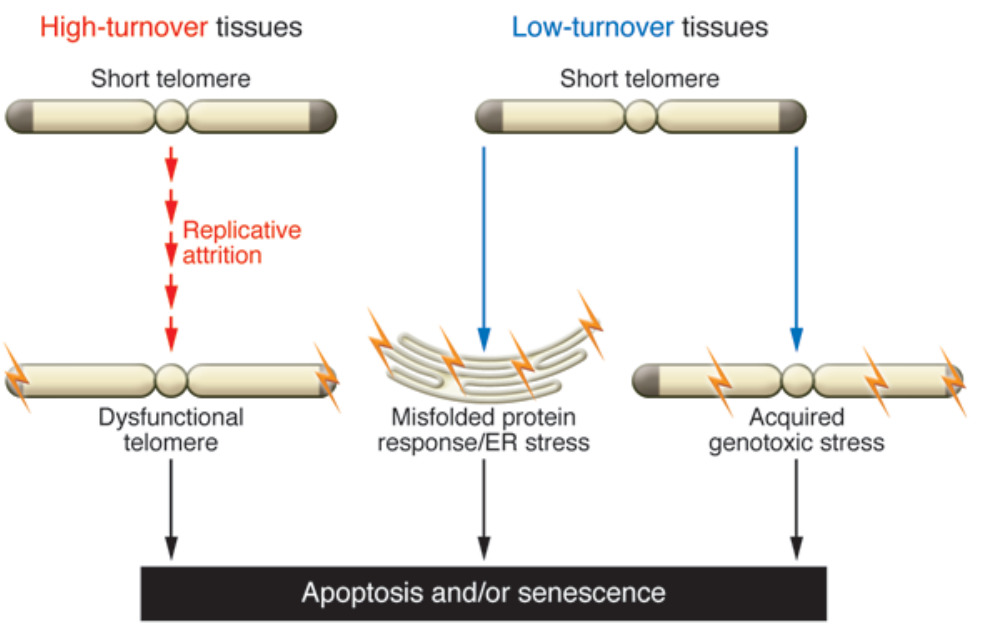

Figure 2

Model for understanding the mechanisms of telomeremediated disease in high- and low-turnover tissues. In high-turnover tissues (left), cell replication is the primary determinant of disease onset. In contrast, in low-turnover tissues (right), other genetic and acquired hits contribute to disease onset. In both cases, telomere dysfunction induces apoptosis and/or senescence. The senescence phenotype may be associated with gene expression changes, mitochondrial dysfunction, aberrant $\mathrm{Ca}^{2+}$ signaling, and the SASP. resent stem cell failure states (45). This framework has important implications for treatment strategies, as discussed below.

\section{Evolving phenotypes in autosomal dominant telomere syndromes}

Telomere length is the primary determinant of disease onset and predominant presentation in telomere disorders. This observation is supported by the fact that in families that carry mutant telomerase genes and display autosomal dominant inheritance, the disease worsens and appears earlier with each successive generation as the telomere length shortens $(12,64)$. Genetic anticipation due to telomere shortening was first recognized in telomerase-null mice, which develop worsening phenotypes with successive breeding (18, $58,65)$. In very late generations, mice die at pre-reproductive ages, which eventually limits the genetic lineage (19). The severity of the genetic anticipation in human families correlates in part with the extent of telomerase loss of function - families with functionally null telomerase alleles show more evident changes in onset across consecutive generations, in contrast to families that carry hypomorphic mutations $(12,35)$. Telomere phenotypes also evolve in autosomal dominant telomere syndromes. In older generations, slow-turnover disease tends to predominate, with IPF being the primary first complication. In later generations, a bone marrow failure-predominant phenotype often comes to attention first (51). Therefore, a single telomerase gene mutation can have heterogeneous manifestations within a given family (51). This evolving pattern is unique to these Mendelian disorders and distinguishes the telomere syndromes from other conditions that show genetic anticipation, such as the trinucleotide repeat expansion syndromes (66). Clinically, this pattern of inheritance poses particular challenges to genetic counseling discussions with at-risk individuals, as the type and onset of disease may be heterogeneous and difficult to predict.

\section{Telomere biology and its role in advancing clinical paradigms}

Telomere-mediated organ failure typically has a protracted course, especially in adults who may have subclinical disease for many years before becoming symptomatic (67). In some cases, for example with an offending insult such as an infection or an exposure to drug toxicities, acute declines can be sustained. In the past, this progressive course has led to a view of telomere disorders (such as aplastic anemia and IPF) as autoimmune processes, and to their empiric treatment with immunosuppression (33). With clear causal links to telomere defects, and with a growing appreciation for the full spectrum of telomere phenotypes, it is now possible to identify affected patients and thus to refine the treatment approach. Patients with telomere-related syndromes are known to have a higher incidence of adverse events with cytotoxic therapies (44) which makes the diagnostic considerations particularly important. The current treatment for telomere-mediated organ failure is primarily supportive, and its complete reversal is feasible only with organ transplantation. Below I highlight some examples in which telomere biology has affected clinical paradigms.

\section{Genetic stratification prior to bone marrow transplant can improve outcome}

Patients with telomere syndromes may have subtle cosmetic features of aging (e.g., premature hair graying), but dysmorphic features are not sensitive and, in our experience, not sufficiently robust to make the diagnosis, even with training. In the setting of bone marrow transplantation, such diagnostic decisions are particularly imperative because patients with telomere syndrome have historically had poor outcomes with conventional bone marrow transplantation (reviewed in ref. 44). Morbidity and mortality occur primarily because of pulmonary and liver toxicity related to chemotherapy used in standard conditioning transplant regimens. With appreciation for the broad telomere-related clinical spectrum, and with the availability of DNA sequencing and telomere length measurement, improved selection has allowed for the testing of reduced-intensity regimens in dedicated studies for patients with telomere disorders. This approach has shown promising short-term outcomes (68).

\section{Considering IPF as a disease of stem cell failure poses a challenge to some current approaches}

IPF treatment is another evolving area in which telomere biology challenges current treatment approaches. IPF is a progressive disorder with a mean survival of 3 years from diagnosis (69). No approved treatments for IPF are currently available, and lung transplantation is accessible to only a small subset of patients who develop end-stage lung disease (less than 5\%) (70,71). Although telomerase mutations are the most commonly identifiable genetic cause for familial pulmonary fibrosis, short telomere length 


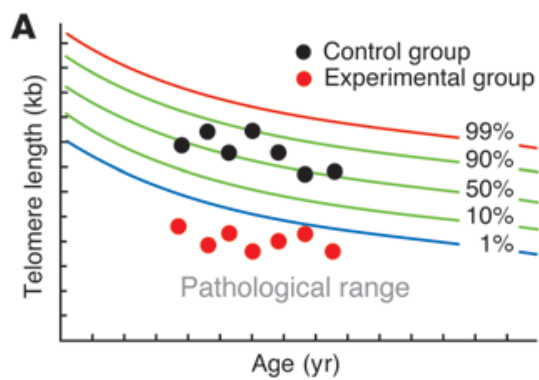

TL difference statistically significant Large absolute TL change

Likely telomere-mediated process

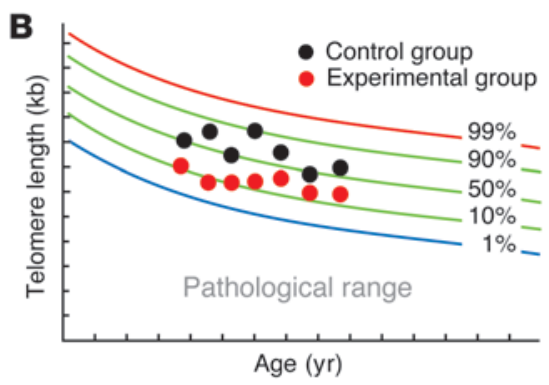

TL difference statistically significant Relatively smaller TL change Less likely telomere-mediated process

\begin{abstract}
Figure 3
Telomere syndromes have defined pathological ranges of telomere shortening. Although short telomere length (TL) has been associated with numerous conditions, in some cases, the shortening reflects acquired replicative stress states rather than telomere-driven degenerative changes. (A) Putative dataset showing large effect size and short telomere length outside of the normal age-adjusted range. (B) Small and statistically significant change in telomere length in hypothetical dataset is less likely to reflect a telomere-mediated process.
\end{abstract}

in pathological ranges is a common feature even in IPF patients without mutations (11). The telomere length defect is likely in the germline, as it is concurrently seen in multiple leukocyte subsets as well as lung epithelial cells (46). This observation has led to the idea that short telomere length may be a risk factor for this disease (46). In support of the idea that telomere length might play a role in driving apparently sporadic IPF is the observation that a subset of IPF patients concomitantly develops cryptogenic liver cirrhosis, another telomere-mediated phenotype (46). In the past two decades, the idea that IPF may be an immune-mediated disease has led to the use of immunosuppressive therapy outside and within clinical trials (ref. 72 and references therein). A recent phase III trial that randomized patients with IPF to the immunosuppressive regimen of $\mathrm{N}$-acetyl cysteine alone, a combination of $\mathrm{N}$-acetyl cysteine, prednisone, and azathioprine, or placebo alone was stopped early because the mortality rate in the group receiving combination treatment was 8-fold higher than that in the placebo group (73). The majority of deaths were reported as respiratory in nature, but it remains unclear whether they were indirectly related to systemic toxicity. Although the role of telomere defects in sporadic forms of IPF is not yet fully understood, the lack of efficacy combined with the increased toxicity seen in recent immunosuppression trials suggests that future clinical approaches to IPF treatment should account for the fact that patients with this form of idiopathic interstitial pneumonia may be exquisitely sensitive to cytotoxic drugs. IPF patients also fare poorly with cancer treatment, an observation that is not commonly noted in patients with other lung disorders (74). It has been suggested that the apparently irreversible scarring pattern of IPF may represent a stem cell failure state that will not amenable to reversal with immunosuppression similar to telomere-mediated aplastic anemia (45). Ultimately, fundamental research in lung biology following the telomere genetic clues has the potential to open paths to new treatment paradigms for age-dependent fibrotic lung disease.

\section{Defining thresholds for pathological telomere shortening in disease contexts}

One important breakthrough that has emerged from the study of human monogenic disorders is the delineation of clinically meaningful thresholds for telomere shortening. Through the use of the telomere length method of flow cytometry and fluorescence in situ hybridization (75), the lymphocyte telomere length in patients with telomere syndrome can be stratified relative to age-matched controls in the population. Early studies that have examined this tool in the monogenic telomere disorders suggest that a thresh- old below the tenth percentile is sensitive, and below the first percentile is fairly specific, for distinguishing individuals who carry mutant telomere genes from their relatives who are noncarriers $(45,46,76)$. These ranges have allowed for the use of this validated method for testing telomere length in the diagnostic work-up of suspected telomere disorders.

The fact that certain age-adjusted thresholds of telomere length have predictive value in clinical settings is significant because short leukocyte telomere length has been associated with numerous disease states and environmental factors, including chronic inflammatory states such as cancer (reviewed in ref. 77), cardiovascular disease (reviewed in ref. 20), and acquired states such as emotional stress, poor socioeconomic status, and education levels (reviewed in refs. 78, 79). Although some of these variables have shown statistically significant telomere shortening consistently across studies, the biological consequences of this relative shortening cannot be equated with the severe telomere length defects seen in the monogenic telomere disorders (Figure 3). While the differences may be statistically significant, the absolute telomere length change in some cases may be small and might therefore reflect acquired replicative stress states rather than telomeredriven degenerative changes such as with the monogenic telomere syndromes. This important caveat should be considered in the interpretation of telomere epidemiology studies.

\section{The role of telomere dysfunction in human cancer}

Clinical observations in patients with telomere syndromes also shed light on the role of telomeres in cancer, which until recently had been primarily studied in cell culture and animal models. Like other DNA repair disorders, telomere disorders are cancer prone; however, the overall incidence is relatively low (80). The cancer-related mortality in patients with telomere syndrome is not known, but it has been estimated that $10 \%$ of patients with dyskeratosis congenita are diagnosed with cancer $(54,80)$. However, that estimate likely includes skin squamous cell cancers, which are prevalent in this group of patients and are usually not lethal (54). Cancers in dyskeratosis congenita have a predilection for high-turnover tissues, with squamous cell carcinomas of the skin and upper aerodigestive tract, myelodysplasia, and acute myeloid leukemia being the most common (80). In a cohort of adults with IPF with TERT mutations, $10 \%$ self-reported a history of cancer, although this rate was not adjusted for age or other exposures (60). These clinical observations make it clear that although telomere syndrome patients are at significantly increased risk for developing cancer, degenerative disease accounts for the majority of the morbidity and mortality in at least $90 \%$ of cases. 
The relatively low overall incidence of cancers in patients with telomere disorders underscores the fact that in the presence of an intact DNA damage response, short telomere length predominantly causes cell loss in humans. These observations are in line with the long-hypothesized role of telomere shortening as a powerful tumor-suppressive mechanism (81). Studies in animal models have shown that short telomeres suppress tumorigenesis by mediating p53-dependent apoptosis and senescence (82). In mice with short telomeres that also lack p53, genomic instability fuels carcinogenesis (83-85). Whether short telomere length in human cancers may contribute to genomic instability at a low level remains a question of ongoing study. Other explanations have been hypothesized to underlie the tumor-prone nature of telomere syndromes, such as compromised immunosurveillance due to the associated immunodeficiency phenotype (22). The stem cell exhaustion state itself has also been proposed to contribute to tumorigenesis, and this would explain the tumor-prone nature of stem cell failure states, such as occurs with non-telomere-mediated aplastic anemia. The clinical study of disease driven by telomere defects provides a unique opportunity to refine current ideas about the role of telomere dysfunction in human cancer development and progression.

\section{Summary and outlook for the future}

Hypothesized molecular mechanisms for aging in modern biology have abounded. These have included stem cell failure, mitochondrial dysfunction, genotoxic stress, and epigenetic changes. Recent cumulative evidence points to telomere shortening as sufficient to provoke all these mechanisms. The manifestations of telomeremediated disease, especially in adults, can be subtle and are often indistinguishable from the slow, gradual functional decline that is a hallmark of aging. The compelling clinical evidence therefore points to telomere shortening itself as being sufficient, or perhaps more broadly representing forms of genotoxic stress that contribute to age-related changes.

In the past decade, telomere biology has provided a molecular rationale for unifying a group of historically considered unrelated disorders under the umbrella of telomere syndromes. The rich, context-dependent clinical presentations of these singlegene disorders and their now appreciated overlap highlight how a molecularly based understanding of disease can refine clinical care at the bedside. This new understanding underscores how the interpretation of increasingly available genetic information might require clinical contextualization before it can be readily applied. Beyond these conceptual considerations, telomere biology has of late brought new tools for diagnosis as well as for understanding disease mechanisms in areas that have long been perplexing to clinicians. Such novel paradigms are particularly needed when it comes to approaching difficult problems such as IPF. The coming years will undoubtedly point to new examples of how the biology of these DNA ends may advance clinical care.

\section{Acknowledgments}

I am particularly indebted to Jonathan Alder for helpful discussions and for assistance with the figures, and to Carol Greider for critical comments on the manuscript. I acknowledge funding support from the NIH (grants R21 HL104345 and RO1 CA160433), the Maryland Stem Cell and Commonwealth Foundations, and the Flight Attendants Medical Research Institute.

Address correspondence to: Mary Armanios, Department of Oncology, Johns Hopkins University School of Medicine, 1650 Orleans St., Cancer Research Building I Room 186, Baltimore, Maryland 21287, USA. Phone: 410.502.3817; Fax: 410.955.0125; E-mail: marmani1@jhmi.edu.
1. Blackburn EH, Greider CW, Szostak JW. Telomeres and telomerase: the path from maize, Tetrahymena and yeast to human cancer and aging. Nat Med. 2006;12(10):1133-1138.

2. Moyzis RK, et al. A highly conserved repetitive DNA sequence, (TTAGGG)n, present at the telomeres of human chromosomes. Proc Natl Acad Sci US A. 1988;85(18):6622-6626.

3. Allshire RC, et al. Telomeric repeat from T. thermophila cross hybridizes with human telomeres. Nature. 1988;332(6165):656-659.

4. Vaziri H, Dragowska W, Allsopp RC, Thomas TE, Harley CB, Lansdorp PM. Evidence for a mitotic clock in human hematopoietic stem cells: loss of telomeric DNA with age. Proc Natl Acad Sci U S A. 1994;91(21):9857-9860.

5. Palm W, de Lange T. How shelterin protects mammalian telomeres. Annu Rev Genet. 2008;42:301-334.

6. Greider CW, Blackburn EH. Identification of a specific telomere terminal transferase activity in Tetrahymena extracts. Cell. 1985;43(2 pt 1):405-413.

7. Greider CW, Blackburn EH. The telomere terminal transferase of Tetrahymena is a ribonucleoprotein enzyme with two kinds of primer specificity. Cell. 1987;51(6):887-898.

8. Greider CW, Blackburn EH. A telomeric sequence in the RNA of Tetrahymena telomerase required for telomere repeat synthesis. Nature. 1989; 337(6205):331-337.

9. Lingner J, Hughes TR, Shevchenko A, Mann M, Lundblad V, Cech TR. Reverse transcriptase motifs in the catalytic subunit of telomerase. Science. 1997; 276(5312):561-567.

10. Feng J, et al. The RNA component of human telomerase. Science. 1995;269(5228):1236-1241.
11. Armanios M. Telomerase and idiopathic pulmonary fibrosis. Mutat Res. 2012;730(1-2):52-58.

12. Armanios M, et al. Haploinsufficiency of telomerase reverse transcriptase leads to anticipation in autosomal dominant dyskeratosis congenita. Proc Natl Acad Sci U S A. 2005;102(44):15960-15964.

13. Dokal I. Dyskeratosis congenita. A disease of premature ageing. Lancet. 2001;(suppl):S27.

14. Harley CB, Futcher AB, Greider CW. Telomeres shorten during ageing of human fibroblasts. Nature. 1990;345(6274):458-460.

15. Allsopp RC, et al. Telomere length predicts replicative capacity of human fibroblasts. Proc Natl Acad Sci U S A. 1992;89(21):10114-10118.

16. Hayflick L, Moorhead PS. The serial cultivation of human diploid cell strains. Exp Cell Res. 1961; 25:585-621.

17. Teixeira MT, Arneric M, Sperisen P, Lingner J. Telomere length homeostasis is achieved via a switch between telomerase- extendible and -nonextendible states. Cell. 2004;117(3):323-335.

18. Hao LY, et al. Short telomeres, even in the presence of telomerase, limit tissue renewal capacity. Cell. 2005;123(6):1121-1131.

19. Armanios M, Alder JK, Parry EM, Karim B, Strong MA, Greider CW. Short telomeres are sufficient to cause the degenerative defects associated with aging. Am J Hum Genet. 2009;85(6):823-832.

20. Aviv A. Genetics of leukocyte telomere length and its role in atherosclerosis. Mutat Res. 2012; 730(1-2):68-74.

21. Goldman F, et al. The effect of TERC haploinsufficiency on the inheritance of telomere length. Proc Natl Acad Sci U S A. 2005;102(47):17119-17124.

22. Armanios M, Blackburn EH. The telomere syn- dromes. Nat Rev Genet. 2012;13(10):693-704.

23. d'Adda di Fagagna F, et al. A DNA damage checkpoint response in telomere-initiated senescence. Nature. 2003;426(6963):194-198.

24. Lee HW, Blasco MA, Gottlieb GJ, Horner JW. Essential role of mouse telomerase in highly proliferative organs. Nature. 1998;392(6676):569-574.

25. Guo N, et al. Short telomeres compromise beta-cell signaling and survival. PloS One. 2011;6(3):e17858.

26. Zhang H, Pan KH, Cohen SN. Senescence-specific gene expression fingerprints reveal cell-type-dependent physical clustering of up-regulated chromosomal loci. Proc Natl Acad Sci US A. 2003;100(6):3251-3256.

27. Coppe JP, et al. Senescence-associated secretory phenotypes reveal cell-nonautonomous functions of oncogenic RAS and the p53 tumor suppressor. PLoS Biol. 2008;6(12):2853-2868.

28. Tchkonia T, Zhu Y, van Deursen J, Campisi J, Kirkland JL. Cellular senescence and the senescent secretory phenotype: therapeutic opportunities. J Clin Invest. 2013;123(3):966-972.

29. Coppe JP, Desprez PY, Krtolica A, Campisi J. The senescence-associated secretory phenotype: the dark side of tumor suppression. Annu Rev Pathol. 2010; 5:99-118.

30. Sahin E, et al. Telomere dysfunction induces metabolic and mitochondrial compromise. Nature. 2011; 470(7334):359-365.

31. Armanios M. Syndromes of telomere shortening. Annu Rev Genomics Hum Genet. 2009;10:45-61.

32. Vulliamy T, et al. The RNA component of telomerase is mutated in autosomal dominant dyskeratosis congenita. Nature. 2001;413(6854):432-435.

33. Yamaguchi $\mathrm{H}$, et al. Mutations in TERT, the gene for telomerase reverse transcriptase, in aplastic ane- 
mia. NEngl J Med. 2005;352(14):1413-1424.

34. Strong MA, Vidal-Cardenas SL, Karim B, Yu H, Guo N, Greider CW. Phenotypes in mTERT/ and $\mathrm{mTERT} /$ mice are due to short telomeres, not telomere-independent functions of telomerase reverse transcriptase. Mol Cell Biol. 2011;31(12):2369-2379.

35. Alder JK, et al. Ancestral mutation in telomerase causes defects in repeat addition processivity and manifests as familial pulmonary fibrosis. PLoS Genet. 2011;7(3):e1001352.

36. Heiss NS, et al. X-linked dyskeratosis congenita is caused by mutations in a highly conserved gene with putative nucleolar functions. Nat Genet. 1998;19(1):32-38.

37. Mitchell JR, Wood E, Collins K. A telomerase component is defective in the human disease dyskeratosis congenita. Nature. 1999;402(6761):551-555.

38. Savage SA, Giri N, Baerlocher GM, Orr N, Lansdorp PM, Alter BP. TINF2, a component of the shelterin telomere protection complex, is mutated in dyskeratosis congenita. Am J Hum Genet. 2008;82(2):501-509.

39. Keller RB, et al. CTC1 mutations in a patient with dyskeratosis congenita. Pediatr Blood Cancer. 2012; 59(2):311-314.

40. Polvi A, et al. Mutations in CTC1, encoding the CTS telomere maintenance complex component 1 , cause cerebroretinal microangiopathy with calcifications and cysts. Am J Hum Genet. 2012;90(3):540-549.

41. Anderson BH, et al. Mutations in CTC1, encoding conserved telomere maintenance component 1 , cause Coats plus. Nat Genet. 2012;44(3):338-342.

42. Knight SW, et al. Unexplained aplastic anaemia, immunodeficiency, and cerebellar hypoplasia (Hoyeraal-Hreidarsson syndrome) due to mutations in the dyskeratosis congenita gene, DKC1. $\mathrm{Br}$ J Haematol. 1999;107(2):335-339.

43. Vulliamy T, Marrone A, Dokal I, Mason PJ. Association between aplastic anaemia and mutations in telomerase RNA. Lancet. 2002;359(9324):2168-2170.

44. de la Fuente J, Dokal I. Dyskeratosis congenita: advances in the understanding of the telomerase defect and the role of stem cell transplantation. Pediatr Transplant. 2007;11(6):584-594.

45. Armanios MY, et al. Telomerase mutations in families with idiopathic pulmonary fibrosis. $N$ Engl J Med. 2007;356(13):1317-1326.

46. Alder JK, et al. Short telomeres are a risk factor for idiopathic pulmonary fibrosis. Proc Natl Acad Sci US A. 2008;105(35):13051-13056.

47. Tsakiri KD, et al. Adult-onset pulmonary fibrosis caused by mutations in telomerase. Proc Natl Acad Sci US A. 2007;104(18):7552-7557.

48. Kirwan $M$, et al. Defining the pathogenic role of telomerase mutations in myelodysplastic syndrome and acute myeloid leukemia. Hum Mutat. 2009; 30(11):1567-1573

49. Calado RT, et al. Constitutional hypomorphic telomerase mutations in patients with acute myeloid leukemia. Proc Natl Acad Sci US A. 2009;106(4):1187-1192.

50. Yamaguchi $\mathrm{H}$, et al. Mutations of the human telomerase RNA gene (TERC) in aplastic anemia and myelo- dysplastic syndrome. Blood. 2003;102(3):916-918

51. Parry EM, Alder JK, Qi X, Chen JJ, Armanios M. Syndrome complex of bone marrow failure and pulmonary fibrosis predicts germline defects in telomerase. Blood. 2011;117(21):5607-5611.

52. Aubert G, Baerlocher GM, Vulto I, Poon SS, Lansdorp PM. Collapse of telomere homeostasis in hematopoietic cells caused by heterozygous mutations in telomerase genes. PLoS Genet. 2012;8(5):e1002696.

53. Jonassaint NL, Guo N, Califano JA, Montgomery EA, Armanios M. The gastrointestinal manifestations of telomere-mediated disease [published online ahead of print December 28, 2012]. Aging Cell. doi:10.1111/acel.12041

54. Dokal I. Dyskeratosis congenita in all its forms. $\mathrm{Br}$ J Haematol. 2000;110(4):768-779.

55. Goldman FD, et al. Characterization of primitive hematopoietic cells from patients with dyskeratosis congenita. Blood. 2008;111(9):4523-4531.

56. Rossi DJ, Bryder D, Seita J, Nussenzweig A, Hoeijmakers J, Weissman IL. Deficiencies in DNA damage repair limit the function of haematopoietic stem cells with age. Nature. 2007;447(7145):725-729.

57. Choudhury AR, et al. Cdkn1a deletion improves stem cell function and lifespan of mice with dysfunctional telomeres without accelerating cancer formation. Nat Genet. 2007;39(1):99-105.

58. Rudolph KL, et al. Longevity, stress response, and cancer in aging telomerase-deficient mice. Cell. 1999;96(5):701-712.

59. Parry EM, et al. Decreased dyskerin levels as a mechanism of telomere shortening in X-linked dyskeratosis congenita. J Med Genet. 2011;48(5):327-333.

60. Diaz de Leon A, et al. Telomere lengths, pulmonary fibrosis and telomerase (TERT) mutations. PLoS One. 2010;5(5):e10680.

61. Calado RT, et al. A spectrum of severe familial liver disorders associate with telomerase mutations. PLoS One. 2009;4(11):e7926.

62. Alder JK, et al. Telomere length is a determinant of emphysema susceptibility. Am J Respir Crit Care Med. 2011;184(8):904-912.

63. Rudolph KL, Chang S, Millard M, Schreiber-Agus $\mathrm{N}$, DePinho RA. Inhibition of experimental liver cirrhosis in mice by telomerase gene delivery. Science. 2000;287(5456):1253-1258.

64. Vulliamy T, Marrone A, Szydlo R, Walne A, Mason PJ, Dokal I. Disease anticipation is associated with progressive telomere shortening in families with dyskeratosis congenita due to mutations in TERC Nat Genet. 2004;36(5):447-449.

65. Blasco MA, et al. Telomere shortening and tumor formation by mouse cells lacking telomerase RNA. Cell. 1997;91(1):25-34.

66. McMurray CT. Mechanisms of trinucleotide repeat instability during human development. Nat Rev Genet. 2010;11(11):786-799.

67. El-Chemaly S, et al. Natural history of pulmonary fibrosis in two subjects with the same telomerase mutation. Chest. 2011;139(5):1203-1209.

68. Dietz AC, et al. Disease-specific hematopoietic cell transplantation: nonmyeloablative conditioning regimen for dyskeratosis congenita. Bone Marrow Transplant. 2011;46(1):98-104

69. King TE Jr, Pardo A, Selman M. Idiopathic pulmonary fibrosis. Lancet. 2011;378(9807):1949-1961.

70. Lama VN. Update in lung transplantation 2008. Am J Respir Crit Care Med. 2009;179(9):759-764.

71. American Thoracic Society, European Respiratory Society. American Thoracic Society/European Respiratory Society International Multidisciplinary Consensus Classification of the Idiopathic Interstitial Pneumonias. This joint statement of the American Thoracic Society (ATS), and the European Respiratory Society (ERS) was adopted by the ATS board of directors, June 2001 and by the ERS Executive Committee, June 2001. Am J Respir Crit Care Med. 2002;165(2):277-304.

72. Raghu G. Idiopathic pulmonary fibrosis: treatment options in pursuit of evidence-based approaches. Eur Respir J. 2006;28(3):463-465.

73. Raghu G, Anstrom KJ, King TE Jr, Lasky JA, Martinez FJ. Prednisone, azathioprine, and $\mathrm{N}$-acetylcysteine for pulmonary fibrosis. $N$ Engl J Med. 2012;366(21):1968-1977.

74. Chida M, Ono S, Hoshikawa Y, Kondo T. Subclinical idiopathic pulmonary fibrosis is also a risk factor of postoperative acute respiratory distress syndrome following thoracic surgery. EurJ Cardiothorac Surg. 2008;34(4):878-881.

75. Baerlocher GM, Vulto I, de Jong G, Lansdorp PM. Flow cytometry and FISH to measure the average length of telomeres (flow FISH). Nat Protoc. 2006; $1(5): 2365-2376$

76. Alter BP, et al. Very short telomere length by flow fluorescence in situ hybridization identifies patients with dyskeratosis congenita. Blood. 2007; 110(5):1439-1447.

77. Prescott J, Wentzensen IM, Savage SA, De Vivo I. Epidemiologic evidence for a role of telomere dysfunction in cancer etiology. Mutat Res. 2012;730(1-2):75-84.

78. Lin J, Epel E, Blackburn E. Telomeres and lifestyle factors: roles in cellular aging. Mutat Res. 2012; $730(1-2): 85-89$.

79. Blackburn EH, Epel ES. Telomeres and adversity: Too toxic to ignore. Nature. 2012;490(7419):169-171.

80. Alter BP, Giri N, Savage SA, Rosenberg PS. Cancer in dyskeratosis congenita. Blood. 2009; 113(26):6549-6557.

81. Greider CW. Cellular responses to telomere shortening: cellular senescence as a tumor suppressor mechanism. Harvey Lect. 2000;96:33-50.

82. Deng Y, Chan SS, Chang S. Telomere dysfunction and tumour suppression: the senescence connection. Nat Rev Cancer. 2008;8(6):450-458.

83. Artandi SE, et al. Telomere dysfunction promotes non-reciprocal translocations and epithelial cancers in mice. Nature. 2000;406(6796):641-645.

84. Chin L, et al. p53 deficiency rescues the adverse effects of telomere loss and cooperates with telomere dysfunction to accelerate carcinogenesis. Cell. 1999;97(4):527-538.

85. Artandi SE, DePinho RA. Telomeres and telomerase in cancer. Carcinogenesis. 2010;31(1):9-18. 